\title{
Entanglement entropy in non-relativistic field theories
}

\section{Sergey N. Solodukhin}

Laboratoire de Mathématiques et Physique Théorique CNRS-UMR 6083, Université de Tours, Parc de Grandmont, 37200 Tours, France

E-mail: solodukh@lmpt.univ-tours.fr

ABSTRACT: We calculate entanglement entropy in a non-relativistic field theory described by the Schrödinger operator. We demonstrate that the entropy is characterized by i) the area law and ii) UV divergences that are identical to those in the relativistic field theory. These observations are further supported by a holographic consideration. We use the non-relativistic symmetry and completely specify entanglement entropy in large class of non-relativistic theories described by the field operators polynomial in derivatives. The entropy of interacting fields is analyzed in some detail. We suggest that the area law of the entropy can be tested in experiments with condensed matter systems such as liquid helium.

Keywords: AdS-CFT Correspondence, Space-Time Symmetries 
Introduction. Entanglement entropy of a field system is defined by tracing over modes that reside inside a chosen surface $\Sigma[1,2]$. If the total system is characterized by a pure quantum state the subsystem inside the surface is described by a density matrix $\hat{\rho}$ with the von Neumann entropy $S=-\operatorname{Tr} \hat{\rho} \ln \hat{\rho}$ known as entanglement entropy (see recent reviews [3, 4] and references therein). This entropy is non-vanishing provided there are short-distance correlations in the system. The presence of such correlations has two consequences: a) entanglement entropy is determined by geometry of the surface $\Sigma$, to leading order by the area of $\Sigma$ and in higher orders by intrinsic and extrinsic geometry of $\Sigma$, see $[5,6]$; and b) the entropy is UV divergent. In d space-time dimensions, for a field system described by a relativistic wave equation, one finds that

$$
S \sim N \frac{\operatorname{Area}(\Sigma)}{\epsilon^{d-2}}
$$

The exact coefficient of proportionality in (1) depends on the regularization scheme, $N$ is the number of field species. Entanglement entropy has been actively studied in the literature. The main focus has been made on the relativistic field systems and especially the conformal field theories. In the latter case a holographic description of entanglement entropy has been proposed that suggests an alternative way to compute the entropy in the strongly coupled conformal field theory dual to supergravity on anti-de Sitter space-time [4].

The definition of entanglement entropy is applicable to any system that has a quantum mechanical description and does not necessarily tie to the Lorentz symmetry. A typical example considered in the literature is a finite system of coupled non-relativistic oscillators $[1,2]$. The Hamiltonian of this system is quadratic in momenta. The continuous limit then reproduces a relativistic field described by the Klein-Gordon operator which is quadratic in time derivative. The primary purpose of the present note is to compute the entanglement entropy for a field system described by the Schrödinger field operator linear in time derivative. The field system in this case can not be presented as a collection of harmonic oscillators. Nevertheless, as we show below, the structure of the entropy in this case is essentially identical to the one in a relativistic field theory.

The AdS/CFT correspondence can be generalized to non-relativistic conformal field theories [7, 8] (for an earlier work see [9]), see also [10]. These theories describe the "fermions at unitarity". In a wider context the non-relativistic theories effectively describe many realistic systems well studied experimentally and theoretically in condensed matter, liquid helium $\mathrm{He}^{4}$ is the standard example. Our results thus are applicable to these systems too.

The field models with the broken Lorentz symmetry are sometimes considered as UV completions of otherwise non-renormalizable relativistic effective theories, e.g. the General Relativity. We anticipate that our results can be extended to those theories.

The replica method. Before proceeding we remind the technical method very useful for calculation of entanglement entropy. This method is known as the replica method, see ref. [11]. One first observes that $-\operatorname{Tr} \hat{\rho} \ln \hat{\rho}=\left.\left(\alpha \partial_{\alpha}-1\right) \operatorname{Tr} \rho^{\alpha}\right|_{\alpha=1}$, where $\hat{\rho}=\frac{\rho}{\operatorname{Tr} \rho}$ is the renormalized density matrix. The next observation is that for the density matrix obtained by tracing over modes inside the surface $\Sigma$ we have that $-\ln \operatorname{Tr} \rho^{\alpha}=W[\alpha]$, 
where $W[\alpha]=-\ln Z(\alpha)$ and $Z(\alpha)$ is the partition function of the field system in question considered on Euclidean space with a conical singularity at the surface $\Sigma$. Thus one has that

$$
S=\left.\left(\alpha \partial_{\alpha}-1\right) W(\alpha)\right|_{\alpha=1}
$$

One chooses the local coordinate system $\left\{X^{\mu}=\left(\tau, x_{i}\right)\right\}$, where $\tau$ is Euclidean time, such that the surface $\Sigma$ is defined by conditions $\tau=0, x_{1}=0$ and $\left(x_{2}, \ldots, x_{d}\right)$ are the coordinates on $\Sigma$. In the subspace $\left(\tau, x_{1}\right)$ it is convenient to choose the polar coordinate system $\tau=$ $r \sin (\phi)$ and $x_{1}=r \cos (\phi)$ where angular coordinate $\phi$ changes in the limits $0 \leq \phi<2 \pi$. The conical space in question is then defined by making the coordinate $\phi$ periodic with the period $2 \pi \alpha$, where $(1-\alpha)$ is very small.

In order to calculate the effective action $W(\alpha)$ we use the heat kernel method. For manifolds with conical singularities this method was earlier developed in great detail in $[12,13]$. Consider a quantum bosonic field described by a field operator $\mathcal{D}$ so that $Z=\operatorname{det}^{-1 / 2} \mathcal{D}$. Then the effective action is defined as

$$
W=-\frac{1}{2} \int_{\epsilon^{2}}^{\infty} \frac{d s}{s} \operatorname{Tr} K
$$

where $\epsilon$ is an UV cut-off, is expressed by means of the trace of the heat kernel $K\left(X, X^{\prime}, s\right)=$ $\left\langle X\left|e^{-s \mathcal{D}}\right| X^{\prime}\right\rangle$ satisfying the heat kernel equation

$$
\begin{aligned}
\left(\partial_{s}+\mathcal{D}\right) K\left(X, X^{\prime}, s\right) & =0, \\
K\left(X, X^{\prime}, s=0\right) & =\delta\left(X, X^{\prime}\right) .
\end{aligned}
$$

In the Lorentz invariant case the heat kernel $K\left(\phi, \phi^{\prime}, s\right)$ (where we skip the coordinates other than angle $\phi)$ on regular flat space depends on the difference $\left(\phi-\phi^{\prime}\right)$. The heat kernel $K_{\alpha}(\phi, \phi, s)$ on space with a conical singularity is then constructed from this quantity by applying the Sommerfeld formula [14]

$$
\begin{aligned}
K_{\alpha}\left(\phi, \phi^{\prime}, s\right)=K(\phi & \left.-\phi^{\prime}, s\right) \\
& +\frac{\imath}{4 \pi \alpha} \int_{\Gamma} \cot \frac{w}{2 \alpha} K\left(\phi-\phi^{\prime}+w, s\right) d w .
\end{aligned}
$$

The contour $\Gamma$ consists of two vertical lines, going from $(-\pi+\imath \infty)$ to $(-\pi-\imath \infty)$ and from $(\pi-\imath \infty)$ to $(\pi-+\imath \infty)$ and intersecting the real axis between the poles of the $\cot \frac{w}{2 \alpha}:-2 \pi \alpha$, 0 and $0,+2 \pi \alpha$ respectively. For $\alpha=1$ the integrand in (5) is a $2 \pi$-periodic function and the contributions of these two vertical lines cancel each other. Thus, for a small angle deficit the contribution of the integral in (5) is proportional to $(1-\alpha)$.

The Laplace operator. In $d$ space-time dimensions, for a relativistic theory described by the massless Klein-Gordon operator or, in Euclidean signature, by the Laplace operator $\nabla^{2}=\partial_{\tau}^{2}+\sum_{i=1}^{d-1} \partial_{i}^{2}$, where $\tau$ is Euclidean time, the heat kernel is known exactly

$$
K\left(\tau, \tau^{\prime}, x, x^{\prime}, s\right)=\frac{1}{(4 \pi s)^{d / 2}} e^{-\frac{1}{4 s}\left[\left(\tau-\tau^{\prime}\right)^{2}+\sum_{i}\left(x_{i}-x_{i}^{\prime}\right)^{2}\right]}
$$


We take $(d-2)$-surface $\Sigma$ to be the plane defined by equations $x_{1}=0, \tau=0$ so that $\left(x_{2}, x_{3}, \ldots, x_{d}\right)$ are coordinates on $\Sigma$. In the polar coordinate system $\tau=r \sin \phi$ and $x_{1}=$ $r \cos \phi$ we have for two points $(r, \phi)$ and $\left(r, \phi^{\prime}\right)$ that $\left(\tau-\tau^{\prime}\right)^{2}+\left(x_{1}-x_{1}^{\prime}\right)^{2}=4 r^{2} \sin ^{2}\left(\frac{\phi-\phi^{\prime}}{2}\right)$. The trace is defined as $\operatorname{Tr} K_{\alpha}=\int d^{d-2} x_{i} \int_{0}^{\infty} d r r \int_{0}^{2 \pi \alpha} d \phi K_{\alpha}\left(\phi=\phi^{\prime}, r^{\prime}=r, x_{i}=x_{i}^{\prime}, s\right)$. For the contour integral $\Gamma$ we find that $[12,13]$

$$
C_{2}(\alpha) \equiv \frac{\imath}{8 \pi \alpha} \int_{\Gamma} \cot \frac{w}{2 \alpha} \frac{d w}{\sin ^{2} \frac{w}{2}}=\frac{1}{6 \alpha^{2}}\left(1-\alpha^{2}\right) .
$$

Thus one obtains for the trace of the heat kernel $([12,13])$

$$
\operatorname{Tr} K_{\alpha}=\frac{1}{(4 \pi s)^{d / 2}}\left(\alpha V+s 2 \pi \alpha C_{2}(\alpha) A(\Sigma)\right),
$$

where $V=\int d \tau d^{d-1} x$ is the volume of spacetime and $A(\Sigma)=\int d^{d-2} x$ is the area of surface $\Sigma$, and

$$
S_{(d)}=\frac{2}{3(d-2)} \frac{\pi}{(4 \pi)^{d / 2}} \frac{A(\Sigma)}{\epsilon^{d-2}}
$$

for entanglement entropy in $d$ space-time dimensions

The Euclidean Schrödinger operator. We consider a non-interacting system described by a non-relativistic field operator $\mathcal{D}=-2 i m \partial_{t}-\sum_{i=1}^{d-1} \partial_{i}^{2}$. Written in this form the Schrödinger operator $\mathcal{D}$ can be viewed as a reduction of the massless Klein-Gordon operator in $d+1$ dimensions $2 \partial_{\xi} \partial_{t}-\partial_{i}^{2}$ after projecting onto the space of fixed momentum $\partial_{\xi}=-i m$ as described in [7-9]. The Euclidean Schrödinger positive operator is further defined as

$$
\mathcal{D}_{E}=2 m \sqrt{-\partial_{\tau}^{2}}-\partial_{i}^{2}
$$

which is formally obtained by a double analytic continuation $t \rightarrow i \tau, m \rightarrow-i m$. The heat kernel of this operator is a product of the heat kernels of two operators

$$
e^{-s \mathcal{D}_{E}}=e^{-s 2 m \sqrt{-\partial_{\tau}^{2}}} \cdot e^{-s\left(-\partial_{i}^{2}\right)}
$$

The heat kernel of the $(d-1)$-dimensional Laplace operator $\partial_{i}^{2}$ is given by expression similar to (6). As for the heat kernel of the first order operator $2 \imath m \partial_{\tau}$ it is convenient first to Laplace transform [15] it to the heat kernel of the second order operator $-\partial_{\tau}^{2}$,

$$
e^{-s 2 m \sqrt{-\partial_{\tau}^{2}}}=\int_{0}^{\infty} d \sigma \frac{m}{\sqrt{\pi}} \frac{s}{\sigma^{3 / 2}} e^{-\frac{s^{2} m^{2}}{\sigma}} e^{-\sigma\left(-\partial_{\tau}^{2}\right)}
$$

and then use expression of the type (6) for the heat kernel of operator $-\partial_{\tau}^{2}$. Switching to the polar coordinates $(r, \phi), \tau=r \sin \phi, x_{1}=r \cos \phi$, we find for the heat kernel function of two points characterized by different angular coodinates $\phi$ and $\phi^{\prime}\left(r^{\prime}=r, x_{i}^{\prime}=x_{i}\right)$,

$$
\left\langle\phi^{\prime}\left|e^{-t \mathcal{D}_{E}}\right| \phi\right\rangle=\frac{2 m s^{2}}{(4 \pi s)^{\frac{d+1}{2}}} \int_{0}^{\infty} \frac{d \sigma}{\sigma^{2}} e^{-(s m)^{2} / \sigma} e^{-r^{2} \sin ^{2} \frac{\chi}{2}\left(\frac{1}{\sigma} \cos ^{2} \frac{\psi}{2}+\frac{1}{s} \sin ^{2} \frac{\psi}{2}\right)},
$$


where we introduced $\chi=\left(\phi-\phi^{\prime}\right)$ and $\psi=\left(\phi^{\prime}+\phi\right)$.

The kernel now depends on two angular variables $\psi$ and $\chi$. This is a consequence of the absence of the Lorentz symmetry. Constructing the heat kernel on space with a conical singularity we have to make the heat kernel to be periodic function of both angles, $\chi$ and $\psi$, with the period $2 \pi \alpha$. We are however interested in the result to leading order in $(1-\alpha)$. The Sommerfeld formula applied to the angular coordinate $\chi$ already gives the term proportional to $(1-\alpha)$. Thus, it is sufficient to apply the formula (5) only to the angular coordinate $\chi$ and consider the other angular variable $\psi$ as periodic with the period $2 \pi$. Calculating the trace one thus first replaces $\chi$ by $\chi+w$, takes $\phi^{\prime}=\phi$, integrates over $\phi$ from 0 to $2 \pi$ and then applies formula (5) to $w$. Using the integral

$$
\int_{0}^{2 \pi} d \phi \int_{0}^{\infty} d r r e^{-r^{2} \sin ^{2} \frac{w}{2}\left(\frac{1}{\sigma} \cos ^{2} \phi+\frac{1}{s} \sin ^{2} \phi\right)}=\frac{\pi \sqrt{s \sigma}}{\sin ^{2} \frac{w}{2}}
$$

and $\int_{0}^{\infty} d \sigma \sigma^{-\nu} e^{-(s m)^{2} / \sigma}=(s m)^{2(1-\nu)} \Gamma(\nu-1)$ and formula (7) we arrive at the expression for the trace of the heat kernel

$$
\operatorname{Tr} e^{-s \mathcal{D}_{E}}=\frac{2 m^{-1} \alpha V}{(4 \pi s)^{\frac{d+1}{2}}}+\frac{s}{(4 \pi s)^{\frac{d}{2}}} 2 \pi C_{2}(\alpha) A(\Sigma),
$$

valid up to terms of order $(1-\alpha)^{2}$. We see that the volume part of the trace of the heat kernel has the dependence on the proper time $s$ typical for the heat kernel of the Laplace operator in $(d+1)$ dimensions. This seems to be consistent with the way we obtained the Schrödinger operator as a reduction from the Klein-Gordon operator in $(d+1)$ dimensions.

On the other hand, rather surprisingly, to leading order in $(1-\alpha)$ the surface term in (13) is precisely identical to the surface term in the heat kernel of the $d$-dimensional Laplace operator (8). The entanglement entropy in a non-relativistic theory in any dimension $d$ is thus given by the same formula (9) as in the case of a real scalar field described by the Lorentz invariant Laplace operator. In four space-time dimensions we hence obtain

$$
S_{(d=4)}=\frac{A(\Sigma)}{48 \pi \epsilon^{2}}
$$

In usual signature the non-relativistic field is naturally described by a complex function. For a complex field everything we have just calculated then should be multiplied by 2 .

The fact that the entropy does not depend on the mass parameter $m$ has a simple explanation. The operator $\mathcal{D}_{E}$ is invariant under the rescaling $\tau \rightarrow \beta \tau, m \rightarrow \beta m$. Note that both terms in (13) are invariant under this rescaling. Since the entropy is not supposed to be a function of $\tau$ (or $t$ in the usual signature) it is not a function of $m$ either.

A holographic consideration. Recently there have been proposed some dual gravity descriptions of non-relativistic systems. In this note we use the approach suggested in [10]. As an example we consider 5-dimensional geometry described by metric

$$
d s^{2}=L^{2}\left(\frac{d r^{2}}{r^{2}}+\frac{1}{r^{2}} \sum_{i=1}^{3} d x_{i}^{2}-\frac{d t^{2}}{r^{2 z}}\right),
$$


which is invariant under the scale transformations

$$
t \rightarrow \lambda^{z} t, x_{i} \rightarrow \lambda x_{i}, r \rightarrow \lambda r
$$

For $z=2$ these are also the symmetries of the Schrödinger operator $\mathcal{D}$. Thus, one expects that (for $z=2$ ) the metric (15) gives us a gravity description of the non-relativistic system. For $z=1$ metric (15) is the anti-de Sitter metric which has larger symmetry $\operatorname{SO}(4,2)$. In this case the AdS/CFT correspondence is at work with a precise dictionary of translation between the quantum field theory and the gravity descriptions.

In the AdS/CFT correspondence it is known that entanglement entropy has a holographic gravity description (for a recent review see [4]). The entropy is defined in the quantum theory living on the boundary of the 5-dimensional space-time (15). Since the geometric quantities are usually divergent when extended till the boundary one considers a regularized boundary at $r=\epsilon$. According to the AdS/CFT dictionary, $\epsilon$ plays the role of the UV regulator on the field theory side. For a given $2 d$-surface $\Sigma$ on the regularized boundary consider a minimal $3 d$-surface $\gamma$ such that it lies entirely on the slice $t=$ const and $\partial \gamma=\Sigma$. According to the holographic proposal the quantity

$$
S_{\mathrm{hol}}=\frac{\operatorname{Area}(\gamma)}{4 G_{5}}
$$

computed on the gravity side with Newton's constant $G_{5}$, is identical to entanglement entropy computed for the quantum fields defined on the $4 d$-boundary of the space-time (15). The correspondence is easily established for the UV divergent part of the entropy as soon as we note that

$$
\operatorname{Area}(\gamma)=\frac{L^{3}}{2 \epsilon^{2}} A(\Sigma)+\ldots
$$

where ... stands for the subleading terms. Moreover, in the AdS/CFT correspondence one has a relation

$$
\frac{\pi L^{3}}{2 G_{5}}=N
$$

where $N$ is the number of fields (species) in the boundary theory. In fact, the theory on the boundary is a $\mathrm{SU}(n)$ Yang-Mills theory, $n=\sqrt{N}$ is the number of colors. Thus, using the holographic proposal one obtains

$$
S_{\mathrm{hol}}=N \frac{A(\Sigma)}{4 \pi \epsilon^{2}}
$$

which is the right expression for entanglement entropy of $N$ relativistic fields in four spacetime dimensions. (Note that the holographic UV regulator $\epsilon$ is not identical to the proper time regulator $\epsilon$ appearing in the heat kernel calculation.)

We now notice that the induced metric on a slice of constant $t$ of (15) does not depend on the parameter $z$. Thus, for a given surface $\Sigma$ on the regularized boundary the minimal surface $\gamma$ and its area are the same for any value of $z$. Assuming that the holographic description of entanglement entropy can be extended to other values of $z$ we obtain that the entropy then is still proportional to the area of $\Sigma$ and is UV divergent in 
the same way as for the relativistic fields. For $z=2$ the theory on the boundary is a non-relativistic theory described by the Schrödinger operator. The holographic description thus is consistent with our direct calculation of entanglement entropy in a field theory with the Schrödinger operator.

Furthermore, from the fact that neither of the quantities that appear in (17) depends on $z$ it is natural to conclude that relation (19) still holds for value $z=2$ so that $N$ in this case is the number of degrees of freedom in the non-relativistic theory. The holographic formula (20) then is consistent with the findings in this note: $N$ non-relativistic fields produce same entanglement entropy as $N$ relativistic fields. Clearly, this statement and the equation (20), can be extended to any dimension $d$. We note that in higher dimension $d$ entanglement entropy is given by expression (9) (the exact numerical prefactor may depend on the regularization scheme) and is invariant under the scale transformations (16). For $z=\frac{3}{2}$ the holographic entropy was earlier calculated in [16] and agrees with our result.

We also note that the invariance under transformations (16) with $\lambda=2$ helps to understand the structure of the heat kernel (13). Under (16) the proper time $s$ rescales in the same manner as $r^{2}, s \rightarrow \lambda^{2} s$. Since the volume $V$ changes as $V \rightarrow \lambda^{d+1} V$ one needs $s^{\frac{d+1}{2}}$ in the denominator of the volume term in (13) for the invariance under transformations (16).

Finally we note that in the holographic description $[7,8]$ of the non-relativistic conformal field theories the metric on the gravity side contains a null circle. This may seem to make more difficult the holographic interpretation of entanglement entropy in this case. However, we observe that the induced metric on the slice $t=$ const in the gravity description of $[7,8]$ is identical to that of in metric (15) so we expect that the holographic description of entanglement entropy considered in the present paper can be extended to the conformal case as well. This however requires a separate more careful consideration.

The interacting fields. So far we have considered the non-relativistic fields without interaction. The holography is however supposed to describe a strongly coupled field system. In order to check the holographic predictions we have to introduce interaction. The interaction can be included by adding a potential term $\int d^{d} x V(\varphi)$ to the classical action, here $\varphi=\left\{\varphi^{i}, i=1, \ldots, N\right\}$ is a set of fields in question.

In the one-loop approximation one splits $\varphi=\varphi_{c}+\varphi_{q}$, where $\varphi_{c}$ is the classical background field and $\varphi_{q}$ is the quantum field. The integration over $\varphi_{q}$ then reduces to calculation of the functional determinant of operator $\mathcal{D}_{E}+M^{2}\left(\varphi_{c}\right)$, where $M_{i j}^{2}=\partial_{i j}^{2} V\left(\varphi_{c}\right)$. The heat kernel of this operator is the product $e^{-s \mathcal{D}_{E}} \cdot e^{-s M^{2}}$. Using the already calculated trace (13) we obtain for the entropy of interacting fields in $d$ dimensions

$$
S_{(d)}=\frac{A(\Sigma)}{12(4 \pi)^{\frac{d-2}{2}}} \operatorname{Tr}\left[M^{d-2} \Gamma\left(1-\frac{d}{2}, M^{2} \epsilon^{2}\right)\right],
$$

where we used that $\int_{\epsilon^{2}}^{\infty} s^{-d / 2} e^{-M^{2} s}=\Gamma\left(1-\frac{d}{2}, M^{2} \epsilon^{2}\right)$. Using the asymptotic behavior $\Gamma(-\alpha, x)=\alpha^{-1} x^{-\alpha}+\ldots$, we find that the leading UV divergence of the entropy (21) is again (multiplied by $N$ ) (9) and is thus not affected by the presence of the interaction in the action. This universal behavior is consistent with the holographic calculation. As we have seen, the holography predicts that the leading UV term in the entropy (20) is the 
same as for free fields. The interaction however shows up in the sub-leading UV divergent and UV finite terms. For instance, in four dimensions we find

$$
S=\frac{A(\Sigma)}{48 \pi}\left(\frac{N}{\epsilon^{2}}+\operatorname{Tr}\left[M^{2}\left(\gamma-1+\ln \left(\epsilon^{2} M^{2}\right)\right)\right]\right)
$$

valid both in relativistic and non-relativistic cases. The second term in (22), depending on the potentieal $V(\varphi)$, may contain the terms subleading in $N$. The matrix $M^{2}$ in $(22)$ depends on the (constant) background field $\varphi_{c}$ so that one may apply the renormalization technique similar to one developed in [17] for cosmic strings. We also notice the earlier work on entanglement entropy in relativistic $O(N)$ model $[18,19]$.

Polynomial field operators, symmetry and the entropy. The symmetry argument helps to obtain the general structure of the entropy in the case when the non-relativistic theory is described by a polynomial field operator

$$
\mathcal{D}_{P}=-\partial_{\tau}^{2}+m^{2(1-n)}\left(-\partial_{i}^{2}\right)^{n} .
$$

The heat operator $\left(\partial_{s}+\mathcal{D}_{P}\right)$ in this case is invariant under the transformations

$$
\begin{aligned}
x_{i} & \rightarrow \lambda x_{i}, & \tau & \rightarrow \lambda^{n} \tau, \\
\text { and } x_{i} & \rightarrow \beta x_{i}, & m & \rightarrow \beta^{\frac{n}{1-n}} m . \lambda^{2 n} s,
\end{aligned}
$$

The trace of the heat kernel then should have the form

$$
\operatorname{Tr}_{\alpha} e^{-s \mathcal{D}_{P}}=a \frac{m^{\frac{(d-1)(n-1)}{n}}}{s^{\frac{d+n-1}{2 n}}} \alpha V+b C_{2}(\alpha)\left(\frac{m^{n-1}}{s^{1 / 2}}\right)^{\frac{d-2}{n}} A(\Sigma),
$$

which is invariant under (24), where $a$ and $b$ are some constants which may depend on $n$ and $d$. Entanglement entropy in a quantum field theory described by the polynomial field operator $(23)$ is

$$
S \sim\left(\frac{m^{n-1}}{\epsilon}\right)^{\frac{d-2}{n}} A(\Sigma)
$$

For $n>1$ the UV divergence of entanglement entropy becomes milder than in the relativistic case (1).

Conclusions. Entanglement entropy is determined by the short-distance correlations in the field system. That's why it is surprising that the non-relativistic theory is characterized by the same entropy as a relativistic field theory although the short-distance behavior of two theories is different. (Indeed, in d dimensions the equal time correlation function is $1 /|\mathbf{x}|^{d-2}$ for a relativistic scalar field and $\delta(\mathbf{x})$ for a non-relativistic field.) In the present note we have demonstrated this by a direct calculation of entanglement entropy using the replica method and by extending the holographic description of entanglement entropy to the non-relativistic theories which have dual gravity descriptions. The agreement between two methods, in particular, indicates that the holographic description of entanglement entropy should have wider applications. 
The fact that we have the area law in the non-relativistic case may have some interesting consequences. In particular, the area law could be checked experimentally. One would have to prepare a pure state in a condensed matter system available at the laboratory and then arrange a situation when part of the system is "hidden" for observations. This may be yet another way to find in condensed matter the features typical for black holes, the analog of the Hawking radiation [20] is the well known other example.

This paper is based on the talk which the author gave at "Symétries non relativistes: théorie mathématique et applications physiques", Tours, 23-24 juin 2009. The helpful discussions with A. Barvinsky, D. Fursaev, O. Lysovyi are kindly acknowledged.

Open Access. This article is distributed under the terms of the Creative Commons Attribution Noncommercial License which permits any noncommercial use, distribution, and reproduction in any medium, provided the original author(s) and source are credited.

\section{References}

[1] L. Bombelli, R.K. Koul, J. Lee and R.D. Sorkin, A quantum source of entropy for black holes, Phys. Rev. D 34 (1986) 373 [SPIRES].

[2] M. Srednicki, Entropy and area, Phys. Rev. Lett. 71 (1993) 666 [hep-th/9303048] [SPIRES].

[3] H. Casini and M. Huerta, Entanglement entropy in free quantum field theory, J. Phys. A 42 (2009) 504007 [arXiv: 0905 .2562] [SPIRES].

[4] T. Nishioka, S. Ryu and T. Takayanagi, Holographic entanglement entropy: an overview, J. Phys. A 42 (2009) 504008 [arXiv:0905.0932] [SPIRES].

[5] S.N. Solodukhin, Entanglement entropy of black holes and AdS/CFT correspondence, Phys. Rev. Lett. 97 (2006) 201601 [hep-th/0606205] [SPIRES].

[6] S.N. Solodukhin, Entanglement entropy, conformal invariance and extrinsic geometry, Phys. Lett. B 665 (2008) 305 [arXiv:0802.3117] [SPIRES].

[7] D.T. Son, Toward an AdS/cold atoms correspondence: a geometric realization of the Schroedinger symmetry, Phys. Rev. D 78 (2008) 046003 [arXiv: 0804.3972] [SPIRES].

[8] K. Balasubramanian and J. McGreevy, Gravity duals for non-relativistic CFTs, Phys. Rev. Lett. 101 (2008) 061601 [arXiv:0804.4053] [SPIRES].

[9] C. Duval, G.W. Gibbons and P. Horvathy, Celestial mechanics, conformal structures and gravitational waves, Phys. Rev. D 43 (1991) 3907 [hep-th/0512188] [SPIRES].

[10] S. Kachru, X. Liu and M. Mulligan, Gravity duals of Lifshitz-like fixed points, Phys. Rev. D 78 (2008) 106005 [arXiv: 0808.1725] [SPIRES].

[11] C.G. Callan, Jr. and F. Wilczek, On geometric entropy, Phys. Lett. B 333 (1994) 55 [hep-th/9401072] [SPIRES].

[12] J.S. Dowker, Quantum field theory on a cone, J. Phys. A 10 (1977) 115 [SPIRES].

[13] D.V. Fursaev, Spectral geometry and one loop divergences on manifolds with conical singularities, Phys. Lett. B 334 (1994) 53 [hep-th/9405143] [SPIRES]. 
[14] A. Sommerfeld, Über verzweigte Potentiale im Raum,, Proc. Lond. Math. Soc. 28 (1897) 395.

[15] I.S. Gradshteyn and I.M. Ryzhik, Table of integrals, series and products, Academic Press, U.S.A. (1980).

[16] T. Azeyanagi, W. Li and T. Takayanagi, On string theory duals of Lifshitz-like fixed points, JHEP 06 (2009) 084 [arXiv: 0905. 0688] [SPIRES].

[17] D.V. Fursaev, The Heat kernel expansion on a cone and quantum fields near cosmic strings, Class. Quant. Grav. 11 (1994) 1431 [hep-th/9309050] [SPIRES].

[18] D.N. Kabat, S.H. Shenker and M.J. Strassler, Black hole entropy in the $O(N)$ model, Phys. Rev. D 52 (1995) 7027 [hep-th/9506182] [SPIRES].

[19] M.A. Metlitski, C.A. Fuertes and S. Sachdev, Entanglement entropy in the $O(N)$ model, arXiv:0904.4477.

[20] O. Lahav et al., A sonic black hole in a density-inverted Bose-Einstein condensate, arXiv:0906.1337. 